\title{
Overcoming Cancer Multidrug Resistance by Codelivery of Doxorubicin and Verapamil with Hydrogel Nanoparticles
}

\author{
Ming Qin, Yong-Eun Koo Lee, Aniruddha Ray, Raoul Kopelman*
}

The efficacy of chemotherapy is often inhibited by multidrug resistance (MDR). A highly engineerable hydrogel nanoparticle (NP) serves as a carrier for the optimal codelivery to tumor cells of the chemodrug, doxorubicin (Dox) and the chemosensitizer, verapamil (Vera), aiming at alleviating tumor MDR. The hydrogel NPs are prepared via the copolymerization of acrylamide and 2-carboxyethyl acrylate. Dox and Vera are post-loaded into the respective NPs, with drug loading around $7.7 \mathrm{wt} \%$ and $8.0 \mathrm{wt} \%$, respectively. The codelivery of Dox-NPs and Vera-NPs increases the intracellular accumulation of Dox, and significantly enhances the cell killing ability of Dox with respect to NCI/ADR-RES cells in vitro. These findings suggest that such codelivery nanoplatforms provide a promising route for overcoming tumor MDR.

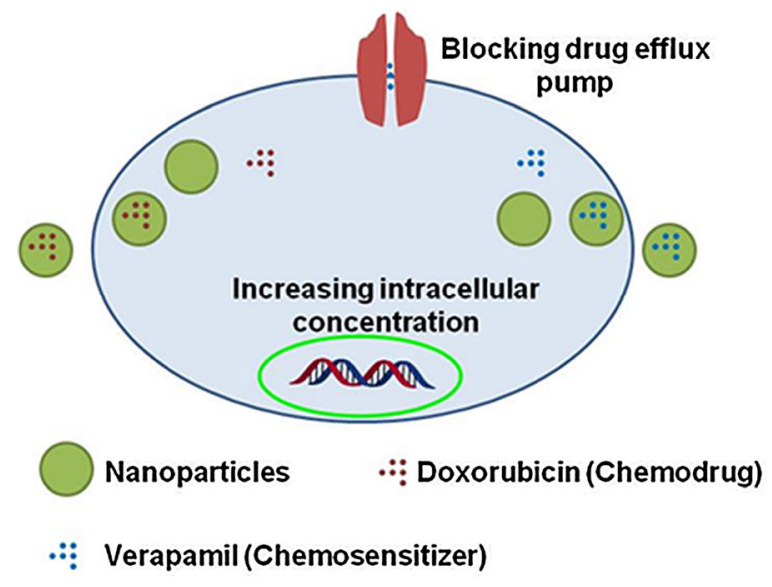

\section{Introduction}

Chemotherapy is one of the main cancer treatment methods. However, its efficacy is often inhibited by multidrug resistance (MDR), a major factor behind aggressive and untreatable disease patterns. MDR is classically defined as a universal state of resilience, against a multiplicity of drugs, including structurally and/or functionally unrelated drugs. ${ }^{[1]}$ The most characterized mechanism of MDR is the drug efflux pump that uses up-regulation of adenosine triphosphate (ATP)-Binding Cassette $(\mathrm{ABC})$ transporters, for example, P-glycoprotein (P-gp). ${ }^{[2]}$ Many chemotherapy drugs, including doxorubicin (Dox), encounter drug resistance. While the drug efflux pump effect can be overcome by increasing the dose, and

Dr. M. Qin, Dr. Y.-E. K. Lee, Dr. A. Ray, Prof. R. Kopelman Department of Chemistry, University of Michigan, Ann Arbor, MI 48109, USA

E-mail: Kopelman@umich.edu thus the concentration of the drug, this may result in unacceptable toxicity. A chemosensitizer, for example, verapamil (Vera), can block the pathway of a drug efflux pump and thus increase the local concentration of the drug in the MDR tumor cell, thereby improving the therapeutic efficacy of the drug. The combination of a chemotherapy drug and a chemosensitizer has been found to be a good option for the treatment of MDR. ${ }^{[2]}$ However, previous clinical trials of chemosensitizers were not quite successful. This was attributed to their poor selectivity and low affinity for P-glycoprotein, thus causing strong side effects. ${ }^{[2-4]}$ For example, a high dose of Vera may cause serious cardiotoxicity. ${ }^{[5]}$ In addition, the dosing and scheduling adjustment of chemotherapy drug and chemosensitizer is challenging because of varying pharmacokinetics, biodistribution and membrane transport considerations. ${ }^{[6]}$

Nano-drug delivery systems (nano-DDS) can prolong the systematic circulation time of drugs, enhance their accumulation in the diseased area, due to multivalent targeting and the enhanced permeability and retention 
(EPR) effect, as well as control of the drug release kinetics, thereby reducing the needed dose, and thus reducing the drug's side effects and enhancing the therapeutic factor. ${ }^{[7,8]}$ Nano-DDS have indeed been shown to alleviate the MDR effect of cancer cells. ${ }^{\left[{ }^{-12]}\right.}$ Nano-DDS may also minimize toxicity due to size-based exclusion from certain organs. ${ }^{[13]}$

Several recent investigations have explored the codelivery of chemotherapy drugs and chemosensitizers with the aid of various nano-DDS, including liposomes, ${ }^{[14-16]}$ solidlipid nanoparticles, ${ }^{[17]}$ micelles ${ }^{[18]}$ and polymeric nanoparticles. ${ }^{[19,20]}$ For example, transferrin (Tf)-conjugated liposomes (Tf-Lip) were used for the codelivery of Dox and Vera. Cell viability tests on Dox-resistant K562 cells, treated with Dox-Vera-Tf-Liposomes, showed 5.2 and 2.8 times greater cytotoxicity [concentration of 50\% inhibition $\left(\mathrm{IC}_{50}\right)=4.18 \times 10^{-6} \mathrm{M}$ ], compared to non-targeted Dox-Vera-liposomes $\left(\mathrm{IC}_{50}=21.7 \times 10^{-6} \mathrm{M}\right)$ and Dox-TfLiposome $\left(\mathrm{IC}_{50}=11.5 \times 10^{-6} \mathrm{M}\right)$, respectively ${ }^{[15]}$ Another study reported the successful encapsulation of Dox and Vera into stealth liposomes. The cytotoxicity tests on MLLB2 rat prostate cancer cells showed that the IC $_{50}$ of Dox-Vera-Liposomes is, respectively, 13 times below that of Dox-liposome + Vera, and 2 times below that of Dox + Vera. ${ }^{[14]}$

One rational guiding this work is that of dosing simplicity, that is, it is easier to change the mix (ratio) of two stock NPs, one containing the drug and the other the sensitizer only, compared to producing a series of NPs loaded with a varying ratio of drug/sensitizer. Another is the use of hydrogel nanoparticles, especially polyacrylamide nanoparticles (PAAm NPs), which have emerged as an important drug delivery vehicle for cancer imaging and therapy. PAAm NPs combine the advantages of hydrogel biocompatibility and the hydrogel NP's engineerability and flexibility, and have enabled multifunctionality, for example, theranostic treatment, controlled release kinetics, stealth circulation and biodegradability. ${ }^{[7,21-23]}$ Notably, this biomaterial, polyacrylamide, has been widely used clinically. For example, it has been used as permanent filler (Aquamid) for facial soft-tissue augmentation for about 20 years. ${ }^{[24]}$ These PAAm NPs are highly soluble in water, and have been made controllably biodegradable; ${ }^{[25]}$ they can carry high payloads of drugs and also protect such drugs from interference by enzymes in the living biological environment, as well as contain surface ligands for the specific targeting of cancer cells. ${ }^{[7]}$ PAAm NPs $(40-100 \mathrm{~nm})$ have been widely used as a delivery vehicle for magnetic resonance imaging, photodynamic therapy, tumor delineation and chemotherapy. ${ }^{[10,22,23,25-29]}$ For example, cisplatin-loaded, F3 peptide-targeted PAAm NPs effectively inhibited the growth of both murine ovarian tumor models and human tumor xenograft models, which was found to be valid not only for cisplatin-sensitive but also for cisplatin-resistant cell lines. ${ }^{[10]}$
Extending our previous work on PAAm NPs, we designed a new kind of hydrogel NP and studied its potential as a codelivery vehicle of Dox and Vera, for overcoming MDR. This kind of NP was prepared via the copolymerization of acrylamide (AAm), 2-carboxyethyl acrylate (CEA) and 3-(acryloyloxy)-2-hydroxypropyl methacrylate (AHM), in a reverse microemulsion system. The novelty of this NP design is in its ability for adjusting the ratio of CEA/AAm in the NP matrix, and this ratio determines the release kinetics of Dox. Either Dox or Vera were post-loaded into the NPs. Compared to the co-embedding of Dox and Vera into the same batch of NPs, the loading of drugs into separate NPs is chosen here because it facilitates dose optimization for both in vitro and in vivo applications. In other words, it is much easier to adjust the ratio of Dox-NPs to Vera-NPs, compared to preparing a series of NPs, each with a different ratio of Dox and Vera. We studied the release kinetics of each drug from the NPs. The NCI/ADR-RES cell line was chosen as a typical example of a Dox-resistant cell line. The accumulation in the NCI/ADR-RES cell line of Dox, from free Dox, Dox-NPs, free Dox + free Vera, and of Dox-NPs + Vera-NPs, was studied by confocal microscopy. The cytotoxicity on the NCI/ADR-RES cell line of either free Dox, Dox-NPs, free Dox + free Vera, Dox-NPs + free Vera, or of Dox-NPs + VeraNPs, was also evaluated. The results demonstrated that the codelivery of Dox-NPs + Vera-NPs can best increase the intracellular accumulation of Dox, as well as significantly improve the cell-killing ability of Dox, on this Dox resistant tumor cell line.

\section{Experimental Section}

\subsection{Materials}

AAm, CEA, AHM, Vera, ammonium persulfate (APS), $N, N, N^{\prime}, N^{\prime}$ tetramethylethylenediamine (TEMED), acrylic acid $N$-hydroxysuccinimide ester (acrylic acid-NHS), sodium dioctyl sulfosuccinate (AOT), Brij 30, dimethylsulfoxide (DMSO), phosphate-buffered saline tablet (PBS), and 3-(4,5-dimethylthiazolyl-2)-2,5-diphenyltetrazolium bromide (MTT) were purchased from Sigma Aldrich. Ethanol (95\%) and hexane were purchased from Fisher Scientific. Dox was purchased from LC laboratories. NCI/ADR-RES cell line was purchased from National Cancer Institute. Hoechst 33342, Roswell Park Memorial Institute medium (RPMI-1640) and 0.05\% TrypsinEDTA (ethylenediamine tetraacetate) were purchased from Invitrogen. Fluorescein-5-thiosemicarbazide (5-FTSC) was purchased from Marker Gene Technologies. All the water used was purified with a Milli-O system from Millipore.

\subsection{Preparation of co(CEA-AAm) NPs}

Deoxygenated hexane (45 mL), AOT (1.6 g) and Brij 30 (4.3 mL) were mixed together, which was stirred vigorously to produce a

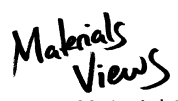

www.MaterialsViews.com 
microemulsion. A mixture of AAm (497 mg), CEA (432 mg) and AHM (428 mg) was dissolved in DI water $(1.3 \mathrm{~mL})$, which was sonicated until dissolved completely. The monomer solution was added into the hexane solution under argon atmosphere. After $20 \mathrm{~min}$, fresh APS solution $(100 \mu \mathrm{L}, 10 \mathrm{wt} \%)$ and TEMED $(100 \mu \mathrm{L})$ were added into the mixture solution to initiate polymerization. After $2 \mathrm{~h}$ reaction, hexane was removed by rotary evaporation. The residue was suspended in ethanol and transferred into an Amicon ultra-filtration cell (Millipore Corp.). In order to remove the surfactants and unreacted monomers, NPs were washed with ethanol and DI water 5 times respectively with a $300 \mathrm{kDa}$ filter membrane under a pressure of 15-20 psi. The NP solution was lyophilized and stored in the freezer. The ratio of CEA/NPs can be adjusted by modifying the amount of monomers added at the beginning of the reaction.

\subsection{Preparation of FITC-labeled NPs}

5-FTSC, acryl acid-NHS (5.6 mg), AAm (497 mg) and Brij 30 (0.1 mL) were dissolved in PBS buffer $(1.3 \mathrm{~mL})$ and kept stirring overnight. Then the solution was mixed with CEA (432 mg) and AHM (428 mg) and sonicated until dissolved completely. Deoxygenated hexane (45 mL), AOT (1.6 g) and Brij 30 (4.3 mL) were mixed together, which was stirred vigorously to produce a microemulsion. The monomer solution was added into the hexane solution under an argon atmosphere. After $20 \mathrm{~min}$, fresh APS solution (100 $\mu \mathrm{L}, 10 \mathrm{wt} \%)$ and TEMED $(100 \mu \mathrm{L})$ were added into the mixture solution to initiate polymerization. After $2 \mathrm{~h}$ reaction, hexane was removed by rotary evaporation. The residue was suspended in ethanol and transferred into an Amicon ultra-filtration cell (Millipore Corp.). In order to remove the surfactants and unreacted monomers, NPs were washed with ethanol and DI water for 5 times respectively with a $300 \mathrm{kDa}$ filter membrane under the pressure of 15-20 psi. The NPs were lyophilized and stored in the freezer.

\subsection{Characterization of NPs}

The size and zeta potential of NPs in aqueous solution were measured with Delsa Nano (Beckman Coulter). Scanning electron microscopy (SEM) images of NPs were obtained with an FEI Nova Nanolab Dualbeam focussed ion beam workstation and scanning electron microscope.

\subsection{Loading of Drugs into NPs}

Both Dox and Vera were loaded into NPs as follows: The NP solution in DI water $\left(10 \mathrm{mg} \mathrm{mL}^{-1}, 1 \mathrm{~mL}\right)$ and a Dox or Vera solution in DI water $\left(10 \mathrm{mg} \mathrm{mL}^{-1}, 20 \mu \mathrm{L}\right)$ were mixed together and kept stirring overnight. Then the drug-loaded NP solution was centrifuged in a centrifuge filter ( $100 \mathrm{kDa}$, Millipore) at $4000 \times g$ for three times, in order to remove the unbound drug molecules. The absorbance of Dox and Vera in NP solution was evaluated using an UV-1601 UVvis spectrometer (Shimadzu). The absorption wavelength of Dox and Vera are $480 \mathrm{~nm}$ and $280 \mathrm{~nm}$, respectively. The concentrations of drugs in the NP solution were calculated from Beer-Lambert Law, while the concentration of the NP solution is known. The loading of drugs into the NPs $(\%)=$ (weight of drug to be loaded/weight of drug-loaded NPs) *100; while the encapsulation efficiency $(\%)=$ (weight of drug found loaded/weight of drug input) * 100 .

\subsection{Drug Release from NPs}

The release kinetics of drugs from NPs was studied under the sink condition, since the concentration of Dox or Vera in our release study was $0.02 \mathrm{mg} \mathrm{mL}^{-1}$, while the solubility of Dox or Vera in PBS was around $10 \mathrm{mg} \mathrm{mL}^{-1}$. Drug-loaded NP solution $\left(1 \mathrm{mg} \mathrm{mL}^{-1}\right.$, $10 \mathrm{ml}$ ) in PBS buffer was prepared and incubated in water bath at $37^{\circ} \mathrm{C}$ for release study. After incubation for $0 \mathrm{~h}, 1 \mathrm{~h}, 3 \mathrm{~h}, 5 \mathrm{~h}, 8 \mathrm{~h}$ and $24 \mathrm{~h}, 1 \mathrm{ml}$ of Drug-NP solution was taken out and transferred into a centrifuge filter $(100 \mathrm{kDa})$. The NP solution was centrifuged at $4000 \times g$ for $15 \mathrm{~min}$ at room temperature, and the filtrate was collected for UV-vis analysis. The factor of Dox degradation was also considered in the release study because of the easy degradation of Dox in PBS buffer. ${ }^{[30]}$ The release profile of Dox was corrected for degradation of Dox.

\subsection{Cell Culture and in Vitro Cytotoxicity Test}

Human ovarian adenocarcinoma cell line NCI/ADR-RES was cultivated in RPMI 1640 medium with 10\% heat-inactivated fetal bovine serum (Hi-FBS). In vitro cytotoxicity of drug-loaded NPs was analyzed in NCI/ADR-RES cell line, using an MTT assay: The cells were incubated with varying formulations of Dox on 96-well plates (5000 cells per well) for $2 d$, in which the final concentration of Dox ranged from 0 to $20 \times 10^{-3} \mathrm{M}$. After that, cells were treated with an MTT reagent solution $\left(0.83 \mathrm{mg} \mathrm{mL}^{-1}\right)$ in colorless RPMI medium for an additional $4 \mathrm{~h}$. Then, the produced formazan crystals were dissolved in DMSO for $1 \mathrm{~h}$. The visible absorption from each well was measured at $550 \mathrm{~nm}$ in a Biochrom Anthos microplate reader.

\subsection{Confocal Microscopy Imaging}

The cells were cultivated on an eight-well chambered cover glass system (Nunc, Lab-Tek) overnight. After that, the cells were incubated with NP solution $\left(1 \mathrm{mg} \mathrm{mL}^{-1}\right)$ for $6 \mathrm{~h}$. Then the Hoechst 33342 dye $\left(1 \mu \mathrm{g} \mathrm{mL}^{-1}\right)$ was added into the cells and incubated with cells for $30 \mathrm{~min}$. After incubation, unbound NPs and Hoechst dye were removed via rinsing with fresh Dulbeccos PBS buffer three times. The cells were incubated in colorless RPMI 1640 medium for the microscopy study, which was done with a Leica confocal microscope (SP-5X) at the Microscopy and Image Analysis Laboratory of the University of Michigan. The fluorescence signal of Dox was excited at $458 \mathrm{~nm}$ and detected over the range of 570$700 \mathrm{~nm}$, while that of FITC-NPs was excited at $488 \mathrm{~nm}$ and the fluorescence was detected over the range of $498-530 \mathrm{~nm}$. Quantitative analysis was performed by comparing the pixel intensity of the fluorescence images using ImageJ.

\subsection{Statistical Analysis}

Results are presented as mean \pm standard deviation from at least three separate experiments. The intracellular uptake and 
<smiles>C=CC(N)=O</smiles>

acrylamide<smiles>C=CC(=O)OCCC(=O)O</smiles>

2-carboxyethyl acrylate<smiles>C=CC(=O)OCC(O)COC(=O)C(=C)C</smiles>

3-(acryloyloxy)-2-hydroxypropyl methacrylate

Scheme 1. Molecular structures of acrylamide (AAm), 2-carboxyethyl acrylate (CEA), and 3-(acryloyloxy)-2-hydroxypropyl methacrylate (AHM).

cyototoxicity of Dox on NCI/ADR-RES cells were evaluated by OneWay Analysis of Variance (ANOVA) followed by Tukey's multiple comparison test using GraphPad Prism v6.00. All analyses were performed with a confidence interval at $95 \%$. $P$-values $<0.05$ were considered significant.

\section{Results and Discussion}

\subsection{Preparation and Characterization of co(CEA-AAm) NPs}

This nanoplatform was prepared by a reverse microemulsion polymerization (Scheme 1), in a monomer mixture droplet containing AAm (main matrix component), AHM (degradable crosslinker) and CEA (monomer providing negatively charged carboxyl groups, uniformly throughout the NP). Cationic drugs (e.g., Dox) can be loaded into negatively charged NPs via coulomb interaction. The mole fraction of CEA was varied from $8 \%$ to $42 \%$ so as to find the optimal CEA concentration that would offer high loading, as well as slow release, of Dox. Results from Dynamic Light Scattering (DLS) analysis showed that the

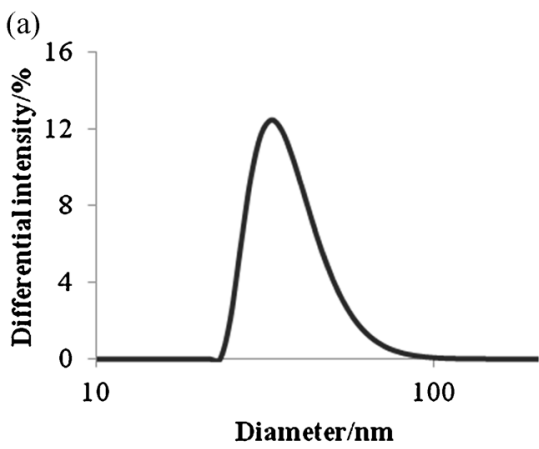

(b)

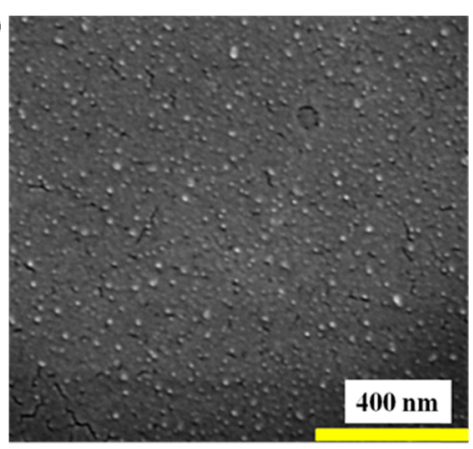

Figure 1. Size distribution of hydrogel NPs from a) DLS and b) SEM image.
Table 1. Size (diameter) and zeta potential of hydrogel NPs from DLS. Data is shown as mean \pm standard deviation.

CEA Size (PBS buffer, Zeta potential

Sample [mol\%] $\quad \mathrm{pH} 7.4)[\mathrm{nm}] \quad$ (DI water) $[\mathrm{mV}]$

$\begin{array}{lccr}\# 1 & 8 & 48 \pm 1 & -49 \pm 9 \\ \# 2 & 25 & 54 \pm 1 & -55 \pm 3 \\ \# 3 & 42 & 74 \pm 3 & -59 \pm 1 \\ \text { Dox-NPs }^{\text {a) }} & 25 & 53 \pm 1 & -50 \pm 3 \\ \text { Vera-NPs }^{\text {b) }} & 25 & 52 \pm 1 & -40 \pm 5\end{array}$

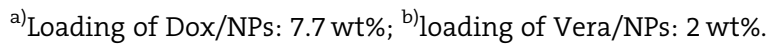

hydrodynamic diameter of NPs ranged from $48.4 \mathrm{~nm}$ to $94.5 \mathrm{~nm}$ in PBS buffer (pH 7.4) (Figure 1a and Table 1). SEM was used to observe the morphology of the dehydrated NPs, which showed an average diameter of around $17 \mathrm{~nm}$ (Figure 1b). The zeta potentials of these hydrogel NPs were around $-50 \mathrm{mV}$ to $-60 \mathrm{mV}$ in DI water (Table 1), which can be attributed to the presence of the carboxyl group in the NPs.

\subsection{Ion Responsive Swelling of co(CEA-AAm) NPs}

We analyzed the size of NPs with varying concentration of $\mathrm{NaCl}$ (from 0 to $1 \mathrm{M}$ ) using DLS (Figure 2), with NPs containing 25\% CEA chosen as example. The results showed that the NP size decreased from $108 \mathrm{~nm}$ to $53 \mathrm{~nm}$ when $\mathrm{NaCl}$ concentration in buffer was increased from $0 \mathrm{M}$ to $0.100 \mathrm{M}$. Further increase in $\mathrm{NaCl}$ concentration from $0.100 \mathrm{~m}$ to $1 \mathrm{~m}$ has no significant impact on the size. These results demonstrate that the NP size was ion concentration-dependent in the range of $0 \mathrm{M}$ to $0.100 \mathrm{M}$. In contrast, the size of PAAm NPs (without any carboxyl group) was stable under varying ion concentrations in the same buffer.

This swelling behaviour is due to the $\mathrm{COO}^{-}$groups in the NPs, which are bound to the matrix and cannot diffuse outside. The counter ions (e.g., $\mathrm{Na}^{+}, \mathrm{Ca}^{2+}$ ), remain confined inside the NPs, to maintain electrical neutrality. Thus the total mobile ion concentration inside the NPs exceeds that of the external solution under lower ion concentration in solution. This causes an osmotic pressure difference and leads to increased water retention. This may be a reason for the NPs to swell in solution with lower ionic strength, and to shrink on increasing the ionic strength. Thus the size of the NP, which is related to the osmotic pressure, depends on the difference between the

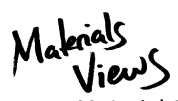

www.MaterialsViews.com 


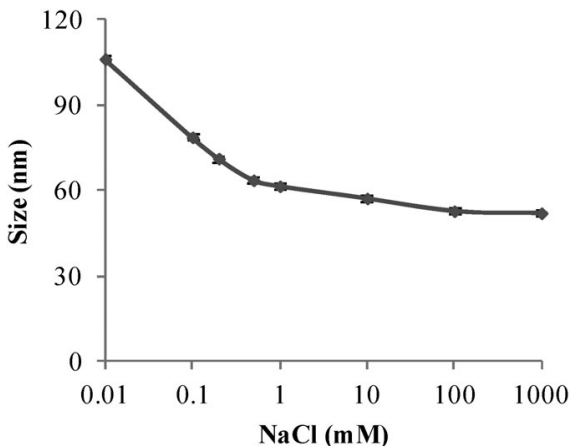

Figure 2. Diameter of hydrogel NPs (from DLS) vs $\mathrm{NaCl}$ concentration in $\mathrm{DI}$ water. Error bars indicate the standard deviations.

mobile ion concentration inside NP matrix and the external solution. $^{[31]}$

\subsection{Loading of Drugs into NPs}

We loaded Dox and Vera into co(CEA-AAm) NPs via postloading, in which the CEA/NPs molar ratio was $8 \%, 25 \%$ and $42 \%$. Dox and Vera were separately loaded into two sets of NPs. The loading of Dox and Vera in the NPs was determined to be $7.7 \mathrm{wt} \%$ and $8.0 \mathrm{wt} \%$ respectively, while the encapsulation efficiency was $81 \%$ and $80 \%$ respectively. DLS analysis results (Table 1 ) showed that the hydrodynamic size of Dox-NPs and Vera-NPs were $53 \pm 1 \mathrm{~nm}$ and $52 \pm 1 \mathrm{~nm}$, respectively, which is similar to the size of blank NPs ( $54 \pm 1 \mathrm{~nm}$ ). After drug loading, the zeta potential of the NPs changed from $-55 \pm 3 \mathrm{mV}$ to $-50 \pm 3 \mathrm{mV}$ (DoxNPs) and $-40 \pm 5 \mathrm{mV}$ (Vera-NPs) respectively, probably due to partial neutralization of the surface charge on the NPs by the Dox and Vera. Additionally we were also able to efficiently incorporate methylene blue, a positively charged photosensitizer, into the co(CEA-AAm) NPs by post loading (data not shown). These results suggested that the co(CEA-AAm) NPs can be used as a high-capacity drug delivery vehicle for cationic drugs. The loading of drugs into NPs can be attributed to non-covalent bonding, especially the strong electrostatic interaction between the anionic NPs and the cationic drug molecules.

\subsection{Degradation of Dox in Dox Solution vs Dox-NP Solution}

Dox easily degrades in PBS buffer at body temperature. We compared the chemical degradation of Dox in free Dox solution and in Dox-NP solution. Figure 3 shows that $37 \%$ of Dox in free Dox solution degraded at $37^{\circ} \mathrm{C}$, in PBS buffer, within $24 \mathrm{~h}$. Over the same time period, only $6 \%$ of Dox in the Dox-NP solution degraded (Figure 3). These results

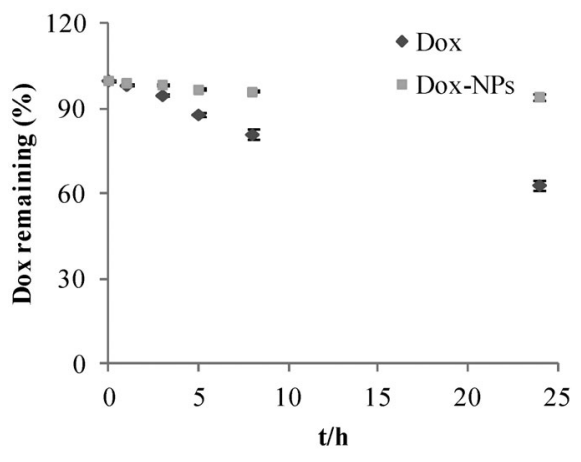

Figure 3. Degradation of Dox in Dox solution and Dox-loaded NP solution in PBS buffer at $37^{\circ} \mathrm{C}$. Dox concentration: $20 \mu \mathrm{g} \mathrm{mg}^{-1}$; Dox/NPs: 2 wt\%. Error bars indicate the standard deviations. indicate that our NPs can effectively slow down the degradation of Dox. Our results are analogous to results from a related publication, ${ }^{[32]}$ which demonstrated that the encapsulation of Dox into polymeric NPs, composed of poly(ethylene glycol) and poloxamer 407 (Pluronic F127), lengthened the lifetime of Dox from $50 \mathrm{~h}$ to $173 \mathrm{~h}$ in PBS buffer. This protective effect can be related to the encapsulation of Dox into the hydrophobic domains of the NPs, reducing the contact of Dox with the hydrophilic environment, which may induce a keto/enol tautomerization and deprotonation of Dox. ${ }^{[33]}$

\subsection{Release Kinetics of Dox and Vera from NPs}

The drug release profile from the NP matrix significantly affects its therapeutic efficacy. We studied the release kinetics of Dox from NPs in which the molar ratio of CEA was fixed at $8 \%, 25 \%$ and $42 \%$. The loading of Dox/NPs was kept at $2 \mathrm{wt} \%$. For analysis of the release study results, the degradation of Dox in PBS buffer was taken into account, using Figure 3 as the calibration curve. It was found that around $20 \%$ of Dox was released within $24 \mathrm{~h}$ from NPs with $42 \%$ CEA; whereas over the same period, around $40 \%$ of Dox was released from NPs with $8 \%$ CEA (Figure 4a). This shows that the release kinetics of Dox from NPs can be adjusted by the NP matrix composition, that is, the mole fraction of CEA. We also studied the release kinetics of Vera from NPs over $24 \mathrm{~h}$, and the loading of Vera/NPs was kept at $2 \%$ wt as well. The molar ratio of CEA in these NPs was fixed at $8 \%, 25 \%$, and $42 \%$. Around $60-$ $70 \%$ of Vera was released over $24 \mathrm{~h}$ from each of the NPs (Figure $4 \mathrm{~b}$ ), demonstrating that the release profile of Vera did not depend on the mole fraction of CEA in the NP matrix.

The release kinetics of drugs from NPs are affected by the property of the matrix and the solubility of the drug in solution. We believe that the release of Dox from NPs is a 

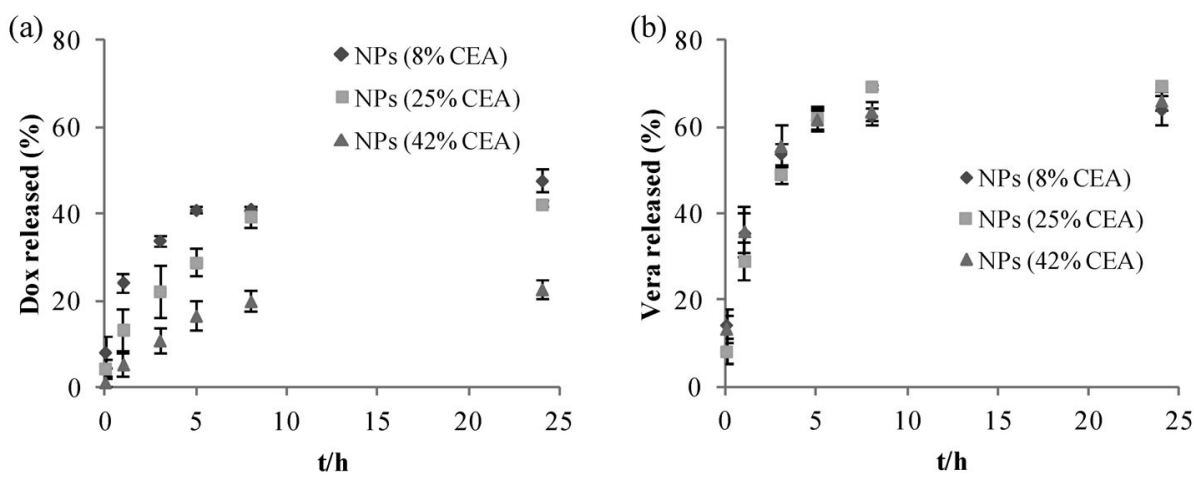

Figure 4. Releasing behaviours of a) Dox and b) Vera from hydrogel NPs with varying ratio of CEA in NP matrix. Dox/NPs: 2 wt\%; Vera/NPs: 2 wt\%; NP concentration: $1 \mathrm{mg} \cdot \mathrm{mL}^{-1}$. Error bars indicate the standard deviations.

diffusion-dominated process. The interaction of Dox with the NP matrix containing $42 \%$ CEA is stronger than that with the NP matrix containing 8\% CEA (more negatively charged as indicated by zeta potential data), causing slower release of Dox from NPs with higher ratios of CEA. However, the release kinetics of Vera from the NPs is independent of the ratio of CEA/NPs, which may be related with the higher hydrophilicity of Vera.

\subsection{Accumulation of Free Dox, Dox-NPs, Free Dox + Free Vera and Dox-NPs + Vera-NPs in NCI/ADR-RES Cells}

We studied the accumulation of free Dox, Dox-NPs, free Dox + free Vera and Dox-NPs + Vera-NPs in cells, using confocal microscopy. ${ }^{[11,34]}$ The human ovarian adenocarcinoma cell line NCI/ADR-RES was chosen because of its reported resistance to Dox. ${ }^{[35]}$ The distribution of Dox in cells was monitored via its own fluorescence signal (red in Figure 5). The cell nucleus was labelled with the Hoechst dyes (blue). Cells were incubated with different formulations of Dox for $6 \mathrm{~h}$. After incubation, unbound drug or NPs were removed by washing with PBS buffer for three times. Our results (Figure 5a) show that, after incubation with free Dox for $6 \mathrm{~h}$, the Dox signal (red) is detected in the NCI/ADRRES cells, showing moderate uptake of Dox into these cells. After incubation with Dox-NPs for $6 \mathrm{~h}$, the intensity of Dox in cells is marginally but not significantly less than that in those cells after incubation with free Dox. After incubation with free Dox + free Vera for $6 \mathrm{~h}$, the signal intensity of Dox in cells was almost six times higher than the fluorescence intensity from cells with just free Dox $(p<0.05)$; after incubation with Dox-NPs + Vera-NPs for the same incubation time, the Dox signal in cells was almost seven times higher than the intensity in cells after incubation with just free Dox (Figure 5b, $p<0.05$ ). The overlap of fluorescence signals from Dox and Hoechst dye (purple in Figure 5a) demonstrated that a large amount of Dox accumulated in the nucleus, while some still remained in the cytoplasm. These results demonstrated that Dox-NPs may not increase the uptake of Dox into tumor cells, compared to uptake from free Dox solution. Similar results were found with polymerlipid hybrid nanoparticles (PLN), which cannot increase the uptake of Dox significantly by MDA435/LCC6/MDR1 human breast cancer cells after $4 \mathrm{~h}$ of incubation. ${ }^{[17]}$ However, most importantly, we found that the formulations of free Dox + free Vera and Dox-NPs + Vera-NPs did significantly improve the uptake of Dox in these drugresistant cells. This confirms that Vera or Vera-NPs, as a chemosensitizer, can inhibit the drug efflux pump and increase the local concentration of Dox in these MDR cells (Scheme 2). ${ }^{[36]}$

\subsection{Colocalization of Dox and FITC-NPs in NCI/ADR-RES Cells}

We also studied the colocalization of Dox molecules and carrier NPs in NCI/ADR-RES cells, via confocal microscopy. In order to track their signal, the NPs were labelled with FITC. Cells were incubated with Dox-FITCNPs and Vera-FITC-NPs for $6 \mathrm{~h}$. As mentioned previously, the Vera-NPs significantly improved the accumulation of Dox in cells. We observed strong fluorescence signals from Dox (red) and the FITC-NP (green), present in the cells (Figure 6a). The green signal from the FITC-NPs inside the cells demonstrates the successful uptake of these NPs into the cells (Figure 6b). However, we also note that the majority of the FITC signal was found on the cell membranes (Figure 6b), indicating that large amounts of NPs may stay attached to the cell membrane. The overlap of the fluorescence signals from Dox and from FITC-NPs in cells is shown in Figure 6c. The colocalization of Dox and FITC-NPs (orange) was found in certain locations in the cells, which may be the location of the Dox-loaded NPs, or of the Dox just released from the NPs. In addition, a strong Dox signal (red) was also

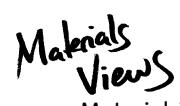

www.MaterialsViews.com 

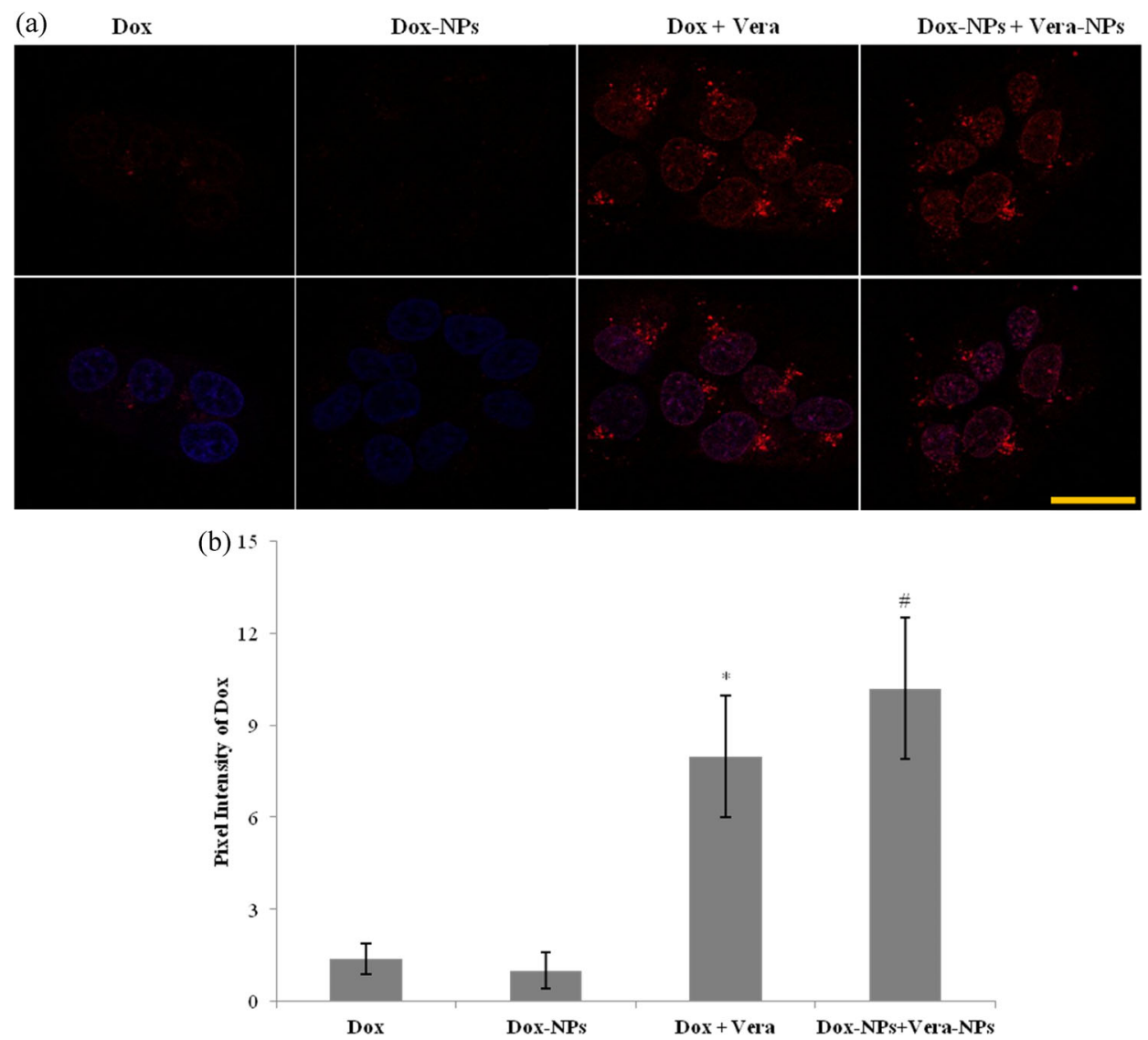

Figure 5. a) Confocal microscopy images of NCI/ADR-RES cells after incubation with free Dox, Dox-NPs, free Dox + free Vera and DoxNPs + Vera-NPs for $6 \mathrm{~h}$. The nucleus is stained with the Hoechst dye (blue). Top: fluorescence signals from Dox (red); bottom: overlap of the fluorescence signals from Dox (red) and Hoechst dye (blue). b) Histogram analysis of pixel intensity of Dox in (a). Dox concentration: $5 \times 10^{-6} \mathrm{M}$; Vera concentration: $5 \times 10^{-6} \mathrm{M}$; scale bar: $25 \mu \mathrm{m}$. Error bars indicate the standard deviations. * $p<0.05$, in comparison to Dox; \# $p<0.05$, in comparison to Dox-NPs and Dox + Vera.

found in other locations inside the cells, showing the intracellular distribution of the Dox that was released from the NPs and delivered into the cells. These results demonstrate that co(CEA-AAm) NPs can be taken up by
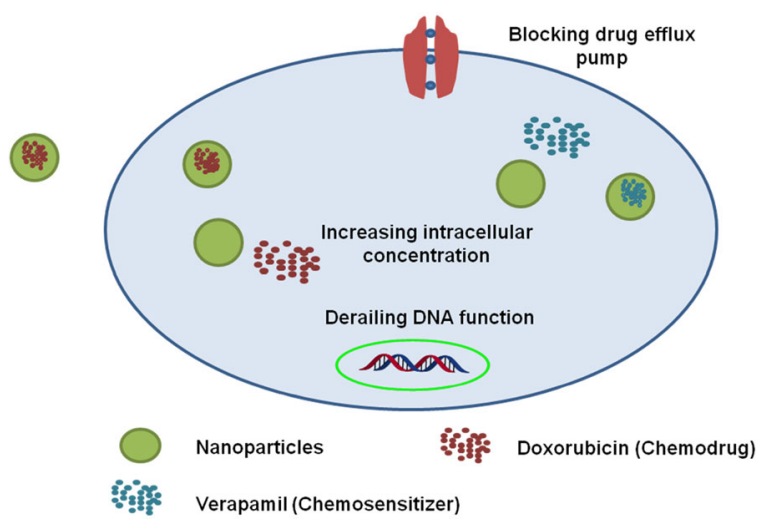

Scheme 2. Delivery into drug-resistant tumor cells of both doxorubicin (derails DNA) and verapamil (blocks efflux pump) with hydrogel NPs. the NCI/ADR-RES cells, and effectively deliver their Dox, which is important for function of the NPs, that is, serving as a drug delivery vehicle.

\subsection{In Vitro Cytotoxicity on NCI/ADR-RES Cells of Free Dox, Dox-NPs, Free Dox + Free Vera, Dox- NPs + Free Vera and Dox-NPs + Vera-NPs}

The cytotoxicity of several formulations of Dox on NCI/ADR-RES cells (Dox-resistant cell line) was studied in vitro using MTT assay. Additionally, we also tested the cytotoxicity of blank NPs and free Vera on the same cell line. The concentration of Vera $\left(5 \times 10^{-6} \mathrm{M}\right)$ was chosen following previous literature. ${ }^{[37]}$ Our results (Figure 7) show that over $90 \%( \pm 7 \%)$ of the cells survived after incubation with blank NPs $\left(1 \mathrm{mg} \mathrm{mL}^{-1}\right)$ for $2 \mathrm{~d}$, while around $98 \%( \pm 8 \%)$ of the cells survived after incubation with free Vera $\left(5 \times 10^{-6} \mathrm{M}\right)$ for $2 \mathrm{~d}$, demonstrating that neither blank NPs nor Vera show significant toxicity to NCI/ADR-RES cells. Then we tested the cytotoxicity of free Dox, Dox-NPs, free Dox + free Vera, Dox-NPs + free Vera 

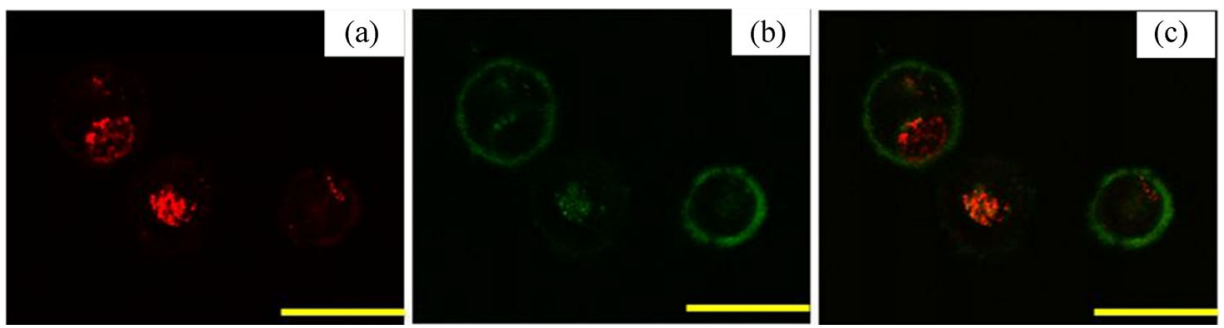

Figure 6. Confocal microscopy images of NCI/ADR-RES cells after $6 \mathrm{~h}$ incubation with Dox-loaded FITC-NPs and Vera-loaded FITC-NPs. a) Fluorescence signal from Dox (red) in the cells; b) fluorescence signal from FITC-NPs (green) in the cells; c) overlap of the fluorescence signal (orange) from Dox and FITC-NPs. Incubation time: $6 \mathrm{~h}$; NP concentration: $1 \mathrm{mg} \mathrm{mL}^{-1}$; scale bar: $25 \mu \mathrm{m}$.

and Dox-NPs + Vera-NPs on the same cell line. Our results (Figure 8 and Table 2) showed that the estimated $\mathrm{IC}_{50}$ of free Dox on NCI/ADR-RES cells was higher than $20 \times 10^{-6} \mathrm{M}$, more than 250 times higher than that on rat gliosarcoma cell line 9L cells $\left(0.07 \times 10^{-6} \mathrm{M}\right)$, confirming that the $\mathrm{NCI} /$ ADR-RES cell line is indeed resistant to Dox. The addition of free Vera reduced the $\mathrm{IC}_{50}$ of Dox to $10 \times 10^{-6} \mathrm{M}(p<0.05)$, which demonstrated that Vera improved the cell killing ability of Dox on this drug-resistant cell line, correlating well with its ability to improve the intracellular accumulation of Dox (see above). The $\mathrm{IC}_{50}$ of Dox-NPs was around $19 \times 10^{-6} \mathrm{M}$, which shows that Dox-NPs have marginally better cell-killing efficiency, compared to free Dox. The $\mathrm{IC}_{50}$ of Dox-NPs + Vera-NPs was found to be around $2.5 \times 10^{-6} \mathrm{M}$, which is 8 times lower than that of the free Dox alone $(p<0.05), 4$ times lower than that of free Dox + free Vera $(p<0.05)$ and marginally but not significantly lower than that of the Dox-NPs + free Vera combination. Notably, the highest concentration of NPs for Dox-NPs + Vera-NPs in Figure 8 was $0.23 \mathrm{mg} \mathrm{mL}^{-1}$, while our previous results (Figure 7) showed that blank NPs were not toxic to cells, even at $1 \mathrm{mg} \mathrm{mL}^{-1}$. Therefore, we believe that the cytotoxicity of Dox-NPs + Vera-NPs (Figure 8) is not due to toxicity of the NP carriers.

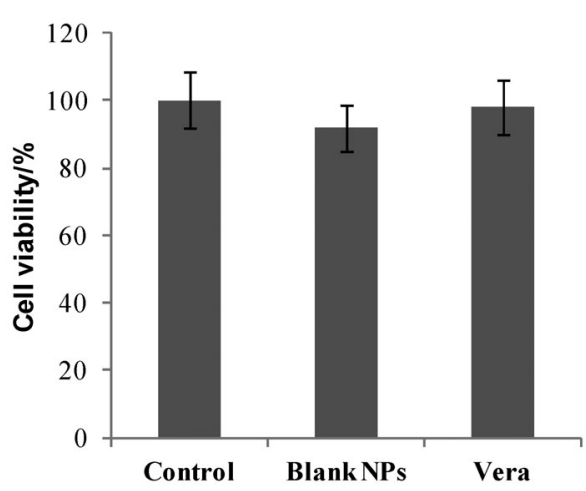

Figure 7. Viability of NCI/ADR-RES cells after incubation with blank NPs $\left(1 \mathrm{mg} \mathrm{mL}^{-1}\right)$ and Vera $\left(5 \times 10^{-6} \mathrm{M}\right)$ for $2 \mathrm{~d}$. Error bars indicate the standard deviations.
The enhanced cell-killing ability of Dox-NPs + Vera-NPs can be attributed to the incorporation of the chemosensitizer (Vera), as well as to the protective effect of the nanoplatform on the Dox. In addition, the NPs may also partially protect Vera from being metabolised, which is mediated by cytochrome P450 enzymes in the NCI/ADR-RES cell line. It has been reported that Vera is subject to an extensive oxidative metabolism mediated by cytochrome P450 enzymes in the body. ${ }^{[38]}$ Indeed, the expression of cytochrome P450 has been observed in the NCI/ADR-RES cell line. ${ }^{[39]}$ Additionally, the encapsulation of Vera into NPs may largely obviate, in vivo, its potential for severe cardiac side effects. ${ }^{[5]}$ This is due to the enhanced accumulation of such Vera-containing NPs into tumors via the EPR effect, and potentially by targeting. ${ }^{[29]}$ In contrast, the much tighter cardiac endothelial walls may prevent any significant local Vera-NP accumulation, as they have been reported to exclude NPs larger than $6 \mathrm{~nm} .{ }^{[13]}$ Thus the use of hydrogel NPs for sensitizer delivery is expected to avoid sensitizer toxicity, while overcoming the MDR effect.

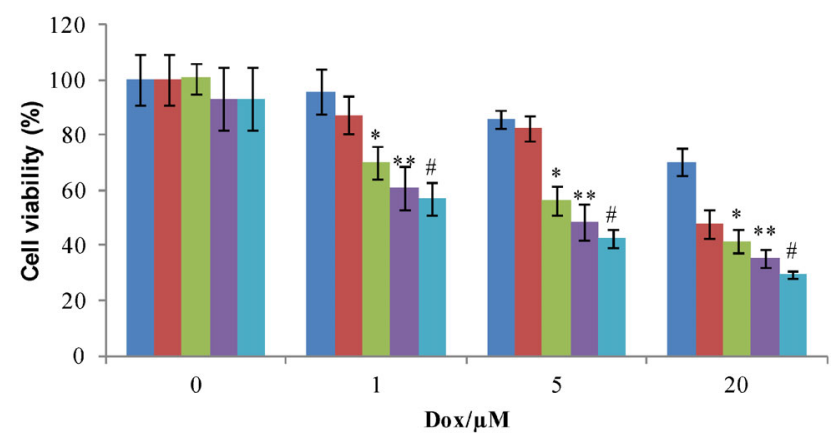

Dox $\square$ Dox-NPs $\square$ Dox +Vera $\square$ Dox-NPs + Vera ${ }^{-D o x}-$ NPs + Vera-NPs

Figure 8. Viability of NCI/ADR-RES cells after incubation for $2 \mathrm{~d}$ with free Dox, Dox-NPs, free Dox + free Vera, Dox-NPs + free Vera and Dox-NPs + Vera-NPs. Dox/NPs: $7.7 \mathrm{wt} \%$; Vera/NPs: 2 wt\%; Vera concentration: $5 \times 10^{-6} \mathrm{M}$. Error bars indicate the standard deviations of seven separate experiments. ${ }^{*} p<0.05$, in comparison to Dox; ${ }^{* *} p<0.05$, in comparison to Dox-NPs; \# $p<0.05$, in comparison to Dox-NPs and Dox + Vera.

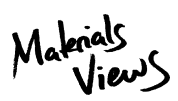

www.MaterialsViews.com 
Table 2. $\mathrm{IC}_{50}$ value of Dox in the formulation of free Dox, Dox-NPs, free Dox + free Vera, Dox-NPs + free Vera and Dox-NPs + Vera-NPs. IC 50

of Dox is the concentration of Dox required to cause $50 \%$ cell killing.

\begin{tabular}{lccccc}
$\begin{array}{l}\text { Estimated IC } \\
{\left[\times \mathbf{1 0}^{-\mathbf{6}} \mathbf{M}\right]}\end{array}$ & of Dox & Dox-NPs & Dox + Vera & Dox-NPs + Vera & Dox-NPs + Vera-NPs \\
\hline NCI/ADR-RES & $>20$ & 19 & 10 & 4.5 & 2.5
\end{tabular}

\section{Conclusion}

With the aim of overcoming MDR, we successfully developed a codelivery system of Dox (chemodrug) and Vera (chemosensitizer) using hydrogel NPs. These hydrogel NPs were prepared via copolymerization of AAm, CEA and AHM, with a varying amount of CEA towards optimized delivery kinetics. We observed that the NP size was ionconcentration dependent. The NP matrix enhances the stability of Dox, and the NP size promises to minimize the cardio-toxicity of Vera, as well as potential Dox toxicity to other organs. These NPs also have a high potential for flexible engineerability, including future specific targeting to the tumor. With this nanoplatform, high loading and slow release of both Dox and Vera have been achieved successfully. The release kinetics of Dox from the NPs is adjustable, based on the ratio of carboxyl groups per NP. This nanoplatform also protects Dox from degradation. Notably, these NP combinations were well incorporated by the Dox-resistant cell line (NCI/ADR-RES), thus behaving as an efficient delivery vehicle for Dox. Moreover, with the aid of free Vera and especially Vera-NPs, the intracellular Dox concentration can be significantly increased, demonstrating the advantage of such a synergistic delivery approach for drug resistant cells. Most importantly, the codelivery of Dox-NPs + Vera-NPs did show a synergistic killing effect on these drug-resistant cells. The IC ${ }_{50}$ of Dox-NPs + Vera-NPs was 8 times lower than that of free Dox alone, or Dox-NPs, and 4 times lower than that of free Dox + free Vera. Thus the codelivery nanoplatform increased the drug efficacy and will potentially minimize toxicity, of both chemodrug and chemosensitizer (due to tumor targeting and size of NP, protecting from cardio-toxicity). It offers a promising nanomedicine approach to drug resistant tumor therapy, and thus should undergo further in vivo studies.

Acknowledgements: The authors thank Teppei Shirakura for discussions on NP preparation, and Leshern Karamchand for the discussions on data analysis. The authors would like to thank James Windak and Paul Lennon at the Chemistry Instrument Shop for their technical support on the UV-vis spectrometer, and the staff of the Electron Microscopy Analysis Laboratory at the University of Michigan for their help on SEM imaging. The authors also thank the staff at the Microscopy and Image Analysis laboratory at the University of Michigan for their help with confocal microscopy imaging.

Received: January 18, 2014; Revised: February 28, 2014; Published online: April 28, 2014; DOI: 10.1002/mabi.201400035

Keywords: cancer therapy; doxorubicin; hydrogel nanoparticles; multidrug resistance; verapamil

[1] M. A. M. Capella, L. S. Capella, J. Biomed. Sci. 2003, 10, 361.

[2] G. Szakacs, J. K. Paterson, J. A. Ludwig, C. Booth-Genthe, M. M. Gottesman, Nat. Rev. Drug Discovery 2006, 5, 219.

[3] E. Crowley, C. McDevitt, R. Callaghan, "Generating Inhibitors of P-Glycoprotein: Where to, Now?", in Multi-Drug Resistance in Cancer, J. Zhou, Ed., Humana Press, Totowa, NJ 2010, p. 405.

[4] A. Palmeira, E. Sousa, M. H. Vasconcelos, M. M. Pinto, Curr. Med. Chem. 2012, 19, 1946.

[5] R. F. Ozols, R. E. Cunnion, R. W. Klecker, T. C. Hamilton, Y. Ostchega, J. E. Parrillo, R. C. Young, J. Clin. Oncol. 1987, 5, 641.

[6] C.-M. J. Hu, S. Aryal, L. Zhang, Therap. Delivery 2010, 1, 323.

[7] Y. E. L. Koo, G. R. Reddy, M. Bhojani, R. Schneider, M. A. Philbert, A. Rehemtulla, B. D. Ross, R. Kopelman, Adv. Drug Delivery Rev. 2006, 58, 1556.

[8] M. E. Davis, Z. Chen, D. M. Shin, Nat. Rev. Drug Discovery 2008, $7,771$.

[9] M. Susa, A. K. Iyer, K. Ryu, F. J. Hornicek, H. Mankin, M. M. Amiji, Z. F. Duan, BMC Cancer 2009, 9, 399.

[10] I. Winer, S. Wang, Y.-E. K. Lee, W. Fan, Y. Gong, D. BurgosOjeda, G. Spahlinger, R. Kopelman, R. J. Buckanovich, Cancer Res. 2010, 70, 8674.

[11] H. Meng, M. Liong, T. Xia, Z. Li, Z. Ji, J. I. Zink, A. E. Nel, ACS Nano 2010, 4, 4539.

[12] A. Khdair, H. Handa, G. Z. Mao, J. Panyam, Eur. J. Pharm. Biopharm. 2009, 71, 214.

[13] M. Gaumet, A. Vargas, R. Gurny, F. Delie, Eur. J. Pharm. Biopharm. 2008, 69, 1.

[14] J. Wang, B. Goh, W. Lu, O. Zhang, A. Chang, X. Y. Liu, T. M. C. Tan, H. Lee, Biol. Pharm. Bull. 2005, 28, 822.

[15] J. Wu, Y. Lu, A. Lee, X. Pan, X. Yang, X. Zhao, R. J. Lee, J. Pharm. Pharmaceut. Sci. 2007, 10, 8.

[16] T. A. Theodossiou, M. C. Galanou, C. M. Paleos, J. Med. Chem. 2008, 51, 6067.

[17] H. L. Wong, R. Bendayan, A. M. Rauth, X. Y. Wu, J. Controlled Release 2006, 116, 275.

[18] L. Fan, F. Li, H. Zhang, Y. Wang, C. Cheng, X. Li, C-h. Gu, O. Yang, H. Wu, S. Zhang, Biomaterials 2010, 31, 5634.

[19] P.-Y. Li, P.-S. Lai, W.-C. Hung, W.-J. Syu, Biomacromolecules 2010, 11, 2576

[20] M.-J. Shieh, C.-Y. Hsu, L.-Y. Huang, H.-Y. Chen, F.-H. Huang, P.-S. Lai, J. Controlled Release 2011, 152, 418. 
[21] Y.-E. K. Lee, R. Kopelman, “Targeted, Multifunctional Hydrogel Nanoparticles for Imaging and Treatment of Cancer: Multifunctional Nanoparticles for Drug Delivery Applications", S. Svenson, R. K. Prud'homme, Eds., Springer, Berlin/ New York 2012, p. 225.

[22] B. Ross, A. Rehemtulla, Y. E. L. Ko, R. Reddy, G. Kim, C. Behrend, S. Buck, R. J. Schneider, M. A. Philbert, R. Weissleder, R. Kopelman, Proc. SPIE 2004, 5331, 76.

[23] R. Kopelman, Y. E. L. Koo, M. Philbert, B. A. Moffat, G. R. Reddy, P. McConville, D. E. Hall, T. L. Chenevert, M. S. Bhojani, S. M. Buck, A. Rehemtulla, B. D. Ross, J. Magn. Magn. Mater. 2005, 293, 404.

[24] N. Pallua, T. P. Wolter, Plast. Reconstruct. Surg. 2010, 125, 1797.

[25] S. Wang, G. Kim, Y.-E. K. Lee, H. J. Hah, M. Ethirajan, R. K. Pandey, R. Kopelman, ACS Nano 2012, 6, 6843.

[26] J. A. Harrel, R. Kopelman, Biophotonics Int. 2000, 7, 22.

[27] H. Xu, S. M. Buck, R. Kopelman, M. A. Philbert, M. Brasuel, B. D. Ross, A. Rehemtulla, Isr. J. Chem. 2004, 44, 317.

[28] G. R. Reddy, M. S. Bhojani, P. McConville, J. Moody, B. A. Moffat, D. E. Hall, G. Kim, Y. E. L. Koo, M. J. Woolliscroft, J. V. Sugai, T. D. Johnson, M. A. Philbert, R. Kopelman, A. Rehemtulla, B. D. Ross, Clin. Cancer Res. 2006, 12, 6677.

[29] G. Nie, H. J. Hah, G. Kim, Y.-E. K. Lee, M. Qin, T. S. Ratani, P. Fotiadis, A. Miller, A. Kochi, D. Gao, T. Chen, D. A.
Orringer, O. Sagher, M. A. Philbert, R. Kopelman, Small 2012, 8, 884.

[30] M. J. H. Janssen, D. J. A. Crommelin, G. Storm, A. Hulshoff, Int. J. Pharm. 1985, 23, 1.

[31] M. Dadsetan, Z. Liu, M. Pumberger, C. V. Giraldo, T. Ruesink, L. Lu, M. J. Yaszemski, Biomaterials 2010, 31, 8051.

[32] D. Missirlis, R. Kawamura, N. Tirelli, J. A. Hubbell, Eur. J. Pharmaceut. Sci. 2006, 29, 120.

[33] J. H. Beijnen, O. A. G. J. van der Houwen, W. J. M. Underberg, Int. J. Pharm. 1986, 32, 123.

[34] F. Shen, S. Chu, A. K. Bence, B. Bailey, X. Xue, P. A. Erickson, M. H. Montrose, W. T. Beck, L. C. Erickson, J. Pharmacol. Exp. Therap. 2008, 324, 95.

[35] L. Wu, A. M. Smythe, S. F. Stinson, L. A. Mullendore, A. Monks, D. A. Scudiero, K. D. Paull, A. D. Koutsoukos, L. V. Rubinstein, M. R. Boyd, R. H. Shoemaker, Cancer Res. 1992, 52, 3029.

[36] L. S. Jabr-Milane, L. E. van Vlerken, S. Yadav, M. M. Amiji, Cancer Treatment Rev. 2008, 34, 592.

[37] R. Sadasivan, R. Morgan, C. Fabian, R. Stephens, Cancer Lett. 1991, 57, 165.

[38] C. Pauli-Magnus, O. von Richter, O. Burk, A. Ziegler, T. Mettang, M. Eichelbaum, M. F. Fromm, J. Pharmacol. Exp. Therap. 2000, 293, 376.

[39] L. J. Yu, J. Matias, D. A. Scudiero, K. M. Hite, A. Monks, E. A. Sausville, D. J. Waxman, Drug Metab. Dispos. 2001, 29, 304. 